\title{
Density-dependent warning coloration
}

$P$ redators learn to avoid unpalatable prey more quickly when it is conspicuous than when it is cryptic ${ }^{1}$. When they are rare, however, conspicuous individuals suffer a greater risk of discovery by predators than the cryptic majority, and their frequency is still too low for predators to learn to avoid them ${ }^{2}$. We might therefore expect selection to favour unpalatable prey that is cryptic at low local densities and conspicuous at high local densities. I have found that such density-dependent warning coloration occurs in Schistocerca emarginata (=lineata) (Orthoptera: Acrididae) grasshoppers, providing insight into the biology of the desert locust, Schistocerca gregaria, and a new perspective on the evolution of warning coloration (aposematism).

Selection on warning coloration by visually hunting predators is predicted to result in monomorphic aposematic prey $^{3}$. Despite this, polymorphisms for aposematism exist ${ }^{4}$. These polymorphic phenotypes represent variations on a similar aposematic theme, rather than a difference between crypsis and conspicuousness. Changes from cryptic to aposematic morphs have been thought of as evolutionary change, not polymorphism, but phenotypic plasticity in coloration can allow this transition to occur within a single generation.

Some populations of juvenile $S$. emarginata in Texas feed mainly on Ptelea trifoliata (Rutaceae), despite the presence of other acceptable host plants, causing nymphs to congregate there. S. emarginata nymphs from populations that feed on Ptelea exhibit a density-dependent colour polymorphism: typically, they are green at low rearing densities, and yellow and black at high rearing densities (Fig. 1a).

Juveniles from a Ptelea-feeding population were reared from hatching outdoors in screen cages as isolated individuals $(n=28)$ or under crowded conditions in groups of ten $(n=31)$. Increased rearing density significantly increased the melanized (black) proportion of the pronotum, wing, femur and abdomen of final-instar insects $(P<0.0001, t$-tests on arcsine-transformed data), as well as the frequency of yellow relative to green and yellow/green background coloration $(P<0.0001$, Monte Carlo contingency table). This polymorphism was independent of rearing $\operatorname{diet}^{5}$.

Yellow-and-black $S$. emarginata were unpalatable to insectivorous lizards (Anolis carolinensis) when reared on Ptelea, but not when reared on Rubus trivialis (Rosaceae) $(P<0.0001$, Fisher's exact test) (Fig. 1b). Unpalatability was mediated by gut content and was rapidly lost when insects were fed a non-toxic plant. All 14 S. emarginata that

Figure 1 Avoidance of Schistocerca emarginata by Anolis carolinensis lizards. a, The green and yellowand-black morphs of S. emarginata. b, Pteleaconferred unpalatability. Adult $A$. carolinensis naive with respect to $S$. emarginata were controlled for motivational state and offered yellow-and-black $S$. emarginata nymphs reared on either Ptelea $(n=19)$ or Rubus $(n=19)$. Consumption without regurgitation was scored as acceptance. Attacking and releasing or regurgitation within 24 hours was scored as rejection. Each lizard was used
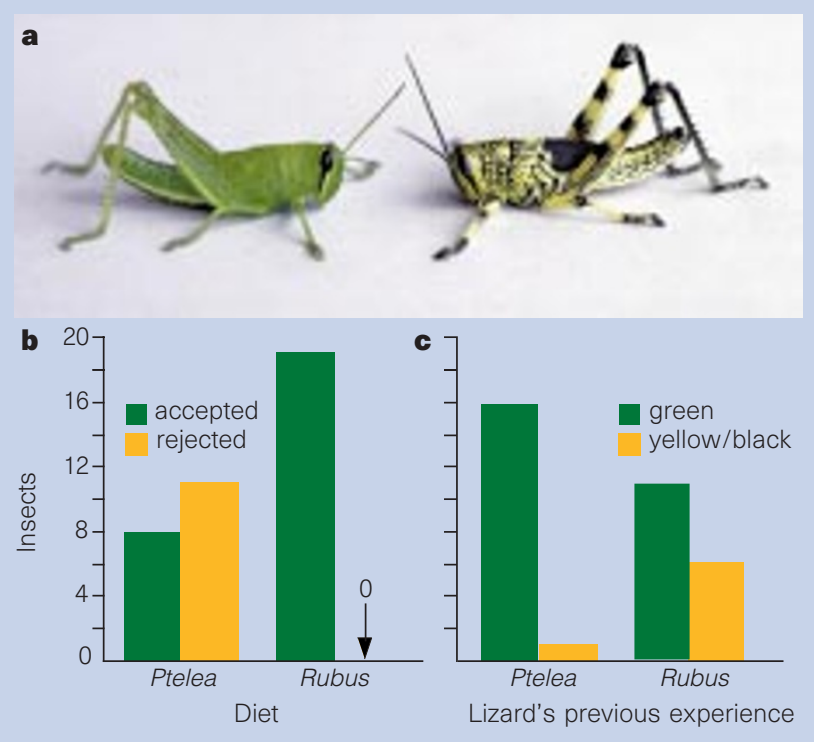

once. c, Lizards discriminated against yellow-and-black in favour of green grasshoppers after experience with yellow-and-black Ptelea-reared grasshoppers. Lizards from the palatability assay were offered a simultaneous-choice test between a green Chortophaga viridifasciata nymph and a similar-sized yellow-and-black S. emarginata nymph. C. viridifasciata were used because it was not possible to rear green $S$. emarginata in sufficient numbers. Lizards eating both insects were omitted from the analysis.

were reared on Ptelea but fed Romaine lettuce for 24 hours were accepted by lizards $(P<0.0001$, Fisher's exact test, comparison with Ptelea-only diet treatment in Fig. 1b). Lizards previously fed unpalatable yellowand-black Ptelea-reared $S$. emarginata discriminated against yellow-and-black grasshoppers in favour of green grasshoppers significantly more often than lizards previously fed palatable yellow-and-black Rubus-reared S. emarginata $(P<0.05$, Fisher's exact test; Fig. 1c). The yellow-andblack coloration of S. emarginata nymphs at high local densities can therefore function as a warning signal when the insects have been feeding on plants containing unpalatable chemicals.

The desert locust, S. gregaria, also exhibits a density-dependent nymphal colour polymorphism ${ }^{6}$, the biological role of which remains a mystery. My results indicate that the colour patterns of $S$. gregaria may be related to specific host plants. In non-outbreak populations, predation can be a strong mortality agent ${ }^{7}$. Nymphs eat plants containing compounds toxic to vertebrates ${ }^{8}$, and crowding on plants can facilitate colour change ${ }^{9}$. Density-dependent aposematism could reduce predation and population extinction rates in areas where insects feed on plants that make them unpalatable to predators, and could hasten the initial stages of population increase under favourable environmental conditions.

Phenotypic plasticity in coloration may have facilitated evolution of aposematism in insects. Density-dependent changes from cryptic to conspicuous phenotypes occur in several insect lineages ${ }^{10}$. Among organisms with the appropriate genotype, densitydependent colour polymorphism could reduce the initial cost of conspicuousness once unpalatability (or some form of unprofitability) has been achieved. The evolution of this trait would be influenced by many factors, including, but not limited to, prey population dynamics and local predator behaviour. Examples of densitydependent aposematism may represent snapshots of this evolutionary process in action.

\section{Gregory A. Sword}

Department of Zoology and Brackenridge Field

Laboratory, University of Texas,

Austin, Texas 78712, USA

Present address: Department of Zoology,

University of Oxford, South Parks Road,

Oxford OX1 3PS, UK

e-mail: greg.sword@zoology.oxford.ac.uk

1. Gittleman, J. L. \& Harvey, P. H. Nature 286, 149-150 (1980).

2. Mallet, J. \& Singer, M. C. Biol. J. Linn. Soc. 32, 337-350 (1987).

3. Fisher, R. A. The Genetical Theory of Natural Selection 2nd edn (Dover, New York, 1958).

4. Endler, J. A. Phil. Trans. R. Soc. Lond. B 319, 505-523 (1988).

5. Sword, G. A. Host Plant Use and Density-Dependent

Aposematism in Schistocerca (Orthoptera: Acrididae). Thesis, Univ. Texas (1998).

6. Stower, W. J. Anti-Locust Bull. 32, 1-75 (1959).

7. Greathead, D. J. J. Appl. Ecol. 3, 239-250 (1966).

8. Bouaïchi, A., Simpson, S. J. \& Roessingh, P. Physiol. Entomol. 21, 247-256 (1996).

9. Ghaout, S., Louveaux, A., Mainguet, A. M., Deschamps, M. \& Rahal, Y. J. Chem. Ecol. 17, 1499-1515 (1991).

10. Fuzeau-Braesch, S. Comp. Insect Physiol. Biochem. Pharmacol. 9, 549-589 (1985). 